\section{PRINCIPIOS PARA UNA ENSEÑANZA DE LA ESCRITURA CREATIVA EN LA UNIVERSIDAD}

\author{
Carlos Peinado Elliot \\ Universidad de Sevilla \\ ORCID iD: https://orcid.org/0000-0002-8469-4120 \\ cpeinado@us.es
}

Cómo citar este artículo/Citation: Peinado Elliot, Carlos (2020). Principios para una enseñanza de la escritura creativa en la universidad. Arbor, 196 (798): a578. https://doi.org/10.3989/ arbor.2020.798n4002

Recibido: 25 mayo 2020. Aceptado: 31 octubre 2020.

RESUMEN: Se antoja necesaria una fundamentación teórica adecuada para una mejor enseñanza de la escritura creativa. El artículo parte de una concepción de la literatura como forma de conocimiento y de la creatividad como proceso sistémico, recupera la imitación y la retórica como fundamentos del oficio, al tiempo que propone posibles vías para una investigación en escritura creativa. Esta se convierte así en un vehículo para la reintegración de los estudios literarios.

PALABRAS CLAVE: Escritura creativa; creatividad; psicología; neurociencia; retórica; imitación; oficio literario.

\section{PRINCIPLES FOR TEACHING CREATIVE WRITING AT UNIVERSITY}

Copyright: (C) 2020 CSIC. Este es un artículo de acceso abierto distribuido bajo los términos de la licencia de uso y distribución Creative Commons Reconocimiento 4.0 Internacional (CC BY 4.0).

ABSTRACT: An adequate theoretical foundation about creativity seems necessary to, subsequently, ask oneself about the most relevant methodology to better teach this subject. The article departs from a systemic conception of creativity, and delves into this based on advances made by psychology and neuroscience, without neglecting other foundations for the development of writing. Creative writing thus becomes a vehicle for the reintegration of literary studies.

KEYWORDS: Creative writing; creativity; psychology; neuroscience; rhetoric; imitation; writing trade. 
No puede resultar extraño que la docencia en escritura creativa (EC) haya surgido en España en las Facultades de Comunicación, pues en ellas convive un interés fuerte por la creatividad junto con la necesidad continua de actualizar los estudios literarios para adaptarse a alumnos cuyo interés principal no es la literatura, pero puede ser el desarrollo de la escritura.

Este espacio fronterizo se presta al desarrollo de una docencia interdisciplinar, que trate de suturar las escisiones que el pensamiento moderno ha causado al conocimiento, confinando lo literario al reducto de las Facultades de Filología. Pero esto no debería ser sino el inicio de una transformación de las propias filologías hacia un modelo de formación más integral. Como ha estudiado Myers, la enseñanza de la escritura en las universidades americanas surge en 1880 como un experimento educativo (Myers, 2006: 4) que persigue reformar y redefinir los estudios académicos de literatura, acercándose a ella como un conocimiento que ha de ser vivido más como un camino de formación que como una ciencia positiva (Bildung frente a Wissenschaft). En este sentido, la EC ha de unir en todo momento conocimiento y práctica. Por ello, los sucesivos modelos que lucharon por instalar la enseñanza de la escritura en la universidad americana se basaron en el trascendentalismo, en una filosofía humanística o abanderaron la llamada pedagogía progresista. El fundador de la EC como disciplina universitaria, Norman Foerster, fue el principal exponente del Nuevo Humanismo y no concibió estos estudios como una disciplina independiente, sino como una rama de estudio en la tradición literaria.

Constantemente se suscita el debate sobre la posibilidad de enseñar creación literaria, pero el hecho de que esta disciplina se imparta desde hace más de un siglo en Estados Unidos, se haya difundido por todo el mundo anglosajón y recientemente haya llegado al ámbito hispanohablante y francófono (Houdart-Merot, 2018) constituye una prueba de la posibilidad real de esta docencia. La resistencia procede de la herencia de la tradición romántica sobre la concepción de la creación literaria -el creador como genio (Royster, 2005: 31-32)-, de ahí que sea necesaria una fundamentación teórica adecuada sobre la creatividad.

\section{LA LITERATURA ES UNA FORMA DE CONOCIMIENTO}

A partir del siglo XIX, una línea de pensamiento ha buscado rehabilitar el arte y la literatura como formas de conocimiento de la realidad. En el siglo XX, la filosofía de Cassirer, al definir las formas simbólicas como funciones «esquematizadoras» mediante las cuales los seres humanos construyen la realidad a través del lenguaje, el arte, el mito, la religión y la ciencia (Barash, 1994: XII), confiere a estas formas una dimensión cognoscitiva y operativa que les otorga una dignidad menoscabada por el pensamiento científicotécnico. Esta línea se ha continuado, por ejemplo, en la revalorización del mito por autores como Blumenberg o la reunificación de la oposición ilustrada entre mito y logos en Hübner o Duch, que presentan mito y ciencia como "formas de pensamiento» que dan razón, desde perspectivas distintas y complementarias, de la realidad.

En esta misma vía, Wilson propone el término consilience, que funde la intuición de las artes con las ciencias (Wilson, 1999: 321-322), al tiempo que propone unir Ciencias Naturales, Sociales y Humanidades, procurando una verdadera sabiduría (1999: 392-393). Los estudios de retórica ya profundizaron en el poder que tienen diversas figuras como vías para el conocimiento de la realidad: así los ya clásicos ensayos sobre la metáfora y la analogía como base de los modelos científicos de Hesse y Black, cuyos logros fueron retomados por Ricoeur (2001). A este mismo filósofo debemos la clarificación de la narración como vía principal para el conocimiento de lo humano (2003) o del simbolismo como forma de conocimiento del mal (2011). Sobre la narración como modelo o metáfora científica ha profundizado también Smorti, quien llega a sostener la existencia de un pensamiento narrativo (Smorti, 2001: 91-94), diferente del paradigmático.

Si la literatura es una forma propia de conocimiento de la realidad, la creación de este conocimiento (no simplemente la interpretación de lo ya descubierto) ha de enseñarse en las aulas, como camino para la investigación simbólica, mítica o narrativa. Así nos acercamos a una concepción completa de la literatura que, en palabras de Quiller-Couch, «is not a mere Science, to be studied; but an ART, to be practised» (Wandor, 2008: 100).

Desde este punto de partida, se nos presentan las concepciones expresivas de los géneros literarios en tanto que formas de percibir lo real y comprendemos elementos constructivos tales como el punto de vista o el narrador como posibilidades de ahondamiento en el mundo a través de la perspectiva, a partir del cual un sujeto (de manera ficticia) se desdobla, tratando de ampliar su modo de percepción. Más aún, los subgéneros implican una concepción de la realidad por parte del escritor (que se formula en una poética), un horizonte de expectativas y una forma de relación con la realidad. En este sentido, las poé- 
ticas no son inocentes (en muchos casos, son inseparables de la ideología) y, por tanto, la pluralidad de subgéneros y de poéticas propicia un enfoque dialógico en la materia que puede abrir posibilidades distintas a los estudiantes.

Por todo ello, esta disciplina ha de ser un camino que se brinda al alumno para adentrarse en un mejor conocimiento, a partir de la palabra, de sí mismo, de los otros, de la sociedad. Como afirma Wandor (2008: 7), EC «is a mode of imaginative thought». La tradición inglesa, ante los ataques recibidos, defiende así que (como las otras artes) la escritura literaria ha de ser enseñada en la universidad (Abbs, 1989: 53-54).

\section{LA CREATIVIDAD COMO FRUTO DE UNA TRIPLE INTERACCIÓN}

Es imprescindible partir de una acertada comprensión del proceso creativo para poder defender y mejorar la enseñanza de la EC en la universidad. Partimos de un modelo integrador o componencial de la creatividad, dentro del cual destacan los de Csikszentmihalyi, Amabile, Feldhusen o Runco y Chand. Estos «tienen en común el reconocer la necesaria pero insuficiente participación de los componentes implicados, y la compleja interacción entre ellos, para que la creatividad sea posible» (Romo, Sanchez-Ruiz y Alfonso-Benlliure, 2017: 58). Como ha observado Csikszentmihalyi (1998: 41), la creatividad es un fenómeno sistémico más que individual, que se compone de tres partes principales (Csikszentmihalyi, 1998: 46): el campo (reglas y procedimientos simbólicos); el ámbito, que incluye a los "guardianes de las puertas que dan acceso al campo» (profesores, editores y críticos); la persona. El proceso creativo abarca los tres componentes: una persona, utilizando los símbolos de un campo, tiene una idea nueva que es sancionada positivamente e incluida por el dominio en el campo a que pertenece. El proceso de enseñanza debe abarcar estos tres aspectos: dominar el campo supone el conocimiento e interiorización de géneros y técnicas de la literatura; el conocimiento del ámbito requiere que el alumno sea consciente también del panorama mediático, académico y editorial que rodea a la creación literaria, al tiempo que ensaya estrategias para actuar en él.

\section{LA CREATIVIDAD EN LA PERSONA: LO QUE LA PSI- COLOGÍA Y LA NEUROCIENCIA NOS DESCUBREN}

\subsection{El proceso creador}

La creación es también un proceso, de modo que la enseñanza habrá de articularse para poder traba- jar sus diferentes etapas. La descripción tradicional de Wallas (preparación, incubación, iluminación y verificación), entendida como proceso recurrente y cíclico, puede aún rastrearse en modelos más recientes y adaptados al ámbito literario. Por ejemplo, para la escritura de guiones, se han señalado tres fases (Bourgeois-Bougrine et al., 2014): impregnación (documentación, lectura, discusión, divagación mental...); estructuración y planificación (elaboración del mapa de la historia); producción, «actual process of writing and rewriting the script» (Bourgeois-Bougrine et al., 2014: 392), donde se unen inspiración y trabajo. Este modelo (concebido como laberinto) abre múltiples caminos entre estas tres amplias etapas, variantes que dependen de factores individuales o situacionales (Bourgeois-Bougrine et al., 2014: 398).

En el ámbito hispánico, Marina (1995: 291) divide el proceso creador en cinco etapas diferentes (proyecto, orden de marcha, ejecución, evaluación, orden de parada), poniendo especial énfasis en la evaluación. El artista debe inventar un «idiocanon», un criterio propio para juzgar, que implica un discernimiento continuo (Marina, 1995: 196). Establecer un «criterio acertado» es la mayor creación del artista (Marina, 1995: 351); formar el gusto es la tarea principal de una educación estética (Marina y Pombo, 2013: 106). De ahí la importancia en la selección de las lecturas que se comentarán en las asignaturas.

\subsection{El cerebro y la creación}

Los avances de la neurociencia han ayudado a profundizar en el funcionamiento del cerebro en los diversos momentos del proceso creativo. En el periodo de incubación la persona debe dejar que la mente vague, para acceder a los procesos mentales inconscientes (Kandel, 2013: 495): mientras que la conciencia se sirve de la atención, que trabaja con pocas opciones, el pensamiento inconsciente abarca una trama inmensa de redes. Dado que gran parte de la memoria es inconsciente, el pensamiento inconsciente es superior para las decisiones que exigen comparar muchas alternativas al mismo tiempo. De ahí la importancia que confiere Marina a "educar el inconsciente» (Marina y Marina, 2013: 120), que tiene una estructura neuronal y cuya sede es la memoria: esta se puede adiestrar mediante la constitución de hábitos, aumentando el arsenal de operaciones que tendrá a su disposición en el proceso inconsciente (pero dirigido) de incubación. Es lo que Martínez-Falero (2020: 159) ha denominado, siguiendo a Gigerenzer, «inteligencia del inconsciente», para la que son cruciales tanto la «memoria textual» como la «ejercitación». 
Junto a ello, aprendemos en este caso la función que cumple la relajación en la preparación de la intuición: cuando las ondas alfa se extienden por el cerebro, es más probable que podamos dirigir el foco de atención hacia el flujo de asociaciones remotas que proceden del hemisferio derecho (Lehrer, 2012: 60) ${ }^{1}$.

Así pues, aunque el momento de la intuición suceda de repente (Lehrer, 2012: 91), es la culminación de anteriores estados cerebrales (Kandel, 2013: 521). En él el hemisferio derecho establece conexiones antes inadvertidas. En la fase preparatoria se activa la corteza visual porque los sujetos dirigen la atención a los datos, centrándose en el problema y bloqueando lo demás (Kandel, 2013: 521). Posteriormente se observa una actividad elevada en el lóbulo temporal derecho justo en el momento en el que se obtiene una solución, lo cual parece probar la importancia del momento de relajación. No obstante, según Schooler no basta con dejar vagar la mente, pues parece conveniente mantener un nivel de conciencia suficiente como para interrumpir este estado y advertir un pensamiento creativo (Lehrer, 2012: 82). De ahí que, como observan Torres-Irribarra, Ibaceta y Preiss (2019: 697), «la divagación mental positiva puede tener una asociación positiva sólo si se presenta de modo adaptativo y no sacando a los individuos por completo de las tareas que deben ejecutar». En todo caso, se constata que la divagación mental intencional es «propia del trabajo creativo» (Torres-Irribarra, Ibaceta y Preiss: 684), así como la divagación mental espontánea (necesaria también en la vida cotidiana).

\section{Como afirma Goldberg,}

Es precisamente la combinación de estos dos procesos, uno de deliberación, guiado por los lóbulos frontales en estado "hiperfrontal», el otro espontáneo y «liberado» del control de los lóbulos frontales en estado hipofrontal, junto con el ir y venir entre estos dos procesos, lo que hace que el proceso creativo sea productivo y finalmente fructífero (Goldberg, 2019: 179).

Por ello, el proceso creativo (Goldberg, 2019: 189) es un "ciclo iterativo" entre hiperfrontalidad e hipofrontalidad. Consecuentemente, en la metodología habrá que entrecruzar actividades que potencien ambas caras de la creación.

En el proceso de escritura, como vemos, se entrecruzan lo consciente y lo inconsciente. Durante el primer borrador, es aconsejable seguir adelante sin tratar de evaluar y evitando la voz interior del censor. Se trata de un proceso cerebral, pues, como han estudiado Berkowitz y Ansari (2008: 542), analizando a través de un escáner a pianistas, en el momento de una improvisación se produce una desactivación de la corteza prefrontal dorsolateral. De ahí que deba procurarse que, entre las actividades propuestas, se encuentren algunas de escritura automática y de improvisación: el alumno ha de aprender a soltarse, aunque debe ser consciente de que la tarea se aplica a una fase concreta del trabajo creativo. En ciertos momentos es necesario evitar la evaluación, pues inhibe las ideas más creativas (Kleinmintz et al., 2014).

La importancia de la fase no-consciente en la creatividad se debe a la configuración de la mente como sistema autoorganizado. Por esta razón el cerebro solo ve lo que está preparado para ver (las pautas existentes) y el simple análisis de una información no aporta nuevas ideas (de Bono, 1994: 38). Es necesario salir de estos caminos preestablecidos para fundar nuevas conexiones (de Bono, 1994: 44). Las actividades que generan este tipo de pensamiento han de ser introducidas en la inventio, como las operaciones provocativas (de Bono).

El proceso de revisión se apoya en un conjunto muy distinto de células que residen en la corteza prefrontal y controlan la atención. La memoria funcional es un instrumento esencial de la imaginación. A veces, lo que se necesita es prestar atención hasta que se entrecrucen los pensamientos necesarios; por ello, es imprescindible proponer una revisión continua del material e iniciar a los alumnos en técnicas de «atención plena». El proceso de revisión se practica a través de la puesta en común en clase (pequeños gruposgrandes grupos), en la revisión con el profesor (tutorías) y entre compañeros (por pares).

Para la revisión final es necesario dejar pasar el tiempo, pues el cerebro contiene dos vías para dar sentido a las palabras y cada una se activa en un contexto diverso. La ruta ventral se activa según Dehaene en pasajes conocidos y tiene como base «una parte de la corteza conocida como área visual de formación de palabras» (Lehrer, 2012: 180). Pero existe otra vía de lectura (franja dorsal), en la que el lector es más consciente de las palabras que aparecen. De ahí que, al leer un pasaje recién escrito, la lectura se realiza por la vía ventral y los errores se hacen invisibles. Al dejar pasar el tiempo, el cerebro se apoya en la franja dorsal, pensando de modo más crítico lo que aparece escrito (Lehrer, 2012: 181-182).

Pero el proceso creativo no es dicotómico: como muestra una investigación dirigida por Beaty et al. (2016: 87), la red cerebral asociada al pensamiento 
espontáneo (default network) y la red cerebral asociada con los procesos cognitivos que requieren atención dirigida externamente (executive control network) cooperan durante el acto creador. En efecto, si bien la pura improvisación puede utilizar la «red neuronal por defecto", una escritura encauzada ya en un impulso formal originario (género, ritmo, metro) o encaminada hacia un objetivo necesitará la cooperación de ambas (Beaty et al., 2016: 88). De hecho, la diferencia entre el escritor experto y el amateur podría encontrarse aquí, según señala Abraham:

In summary, then, while creative writing in general engages regions of the $\mathrm{DMN}$ and the semantic control network (SCN), expert writers additionally recruit the central executive network (CEN) during creative writing (Abraham, 2018: 221).

Por otra parte, también la revisión puede incluir la «red neuronal por defecto» (Beaty et al., 2016: 9192). En general, esta influye en la creación de ideas que la "red neuronal orientada a tareas» puede limitar y dirigir a fines específicos (Beaty et al., 2016: 92). De ahí que, en un artículo posterior, formule la actividad creativa como un proceso que involucra todo el cerebro (Beaty et al., 2018: 1087). Por ello, debemos establecer actividades de diverso tipo, que puedan poner en juego las diferentes redes neuronales.

\subsection{Personalidad creadora}

Como afirman Romo, Sanchez-Ruiz y Alfonso-Benlliure (2017: 58), todos los modelos integradores de la creatividad (basados en la interacción de diversos componentes) «incluyen la personalidad, o algún aspecto de esta, como uno de esos componentes clave». De los distintos modelos teóricos de personali$\mathrm{dad}^{2}$, tiene un mayor respaldo el de los cinco grandes, que cifra como factores de la personalidad los siguientes: apertura a la experiencia, responsabilidad, extraversión, amabilidad y neuroticismo. Como indica Romo (2019: 252-255), solo el primero (apertura a la experiencia) se relaciona de una manera clara con el carácter creador: «imaginación, fantasía, sensibilidad estética, atención a los sentimientos internos, preferencia por la variedad, curiosidad intelectual y valores no convencionales». No obstante, también hay elementos del segundo que pueden relacionarse con el carácter creador: "autodisciplina, sentido del deber, confianza, esfuerzo por el logro, preferencia por la conducta organizada y planificada, baja impulsividad». En este sentido, Marina (1995: 193) subraya la importancia de la tenacidad: el proyecto no es solo una visión inicial, y tiene que afrontar numerosas di- ficultades (Gardner, 2005: 449). Es interesante observar cómo estos rasgos coinciden con los factores clave en el desarrollo de la EC, según el estudio realizado por Barbot et al. (2012: 218): «observation, generation of description, imagination, intrinsic motivation and perseverance».

Piirto (2009: 3-4) resume los rasgos indicados por los principales estudios: androginia, creatividad, imaginación, visión, intuición, introversión, apertura a la experiencia, sobreexcitabilidad, pasión por trabajar en un área, perceptividad, resiliencia, tolerancia a la ambigüedad, preferencia por la complejidad, persistencia, voluntad, autodisciplina, autoeficacia, capacidad de arriesgarse. Manuela Romo (2019: 259-266) también cree preferible acercarse a la personalidad del creador a partir de algunos rasgos destacados.

No hay, como se puede ver, una única forma de ser creativo. Así, el modelo de doble vía (Baas et al., 2013) muestra una función de flexibilidad cognitiva junto a otra de persistencia cognitiva. Algunos rasgos influyen en la creatividad por su relación con la flexibilidad (capacidad de cambiar de perspectiva), mientras que otros por estar vinculados con la persistencia (esfuerzo y atención focalizada). Los primeros serían extroversión, apertura a la experiencia, motivación y afectividad positiva, mientras que estarían entre los segundos afectividad negativa o neuroticismo (Baas et al., 2013: 735-737).

De todo lo anterior se deduce la importancia que tiene realizar un camino de fortalecimiento y liberación personal en el artista (cfr. el método de Julia Cameron), la necesidad de cultivar en las clases y tareas actitudes de trabajo, observación, curiosidad y revisión continua. Esto ha de ser tenido en cuenta para la evaluación de la asignatura. No es extraño que, para algunos fundadores de la EC (como Briggs), el objetivo de la instrucción en escritura fuera hacer el carácter moral más eficiente a través de la escritura diaria a partir de un cultivo deliberado de la percepción (Myers, 2006: 54). Igualmente, es necesario proponer actividades diversas y flexibles, de modo que cada uno pueda encontrar su propio camino, así como para no causar alteraciones radicales en los procesos de escritura de los estudiantes.

\subsection{Interrelación e interdisciplinariedad. Importancia de espacios y ambientes creativos}

Como ha analizado Andreasen (2005: 109-132), junto con los factores hereditarios hay componentes ambientales que favorecen el cultivo de la creatividad. Los estudios de Allen muestran que son los empleados con 
mayor número de interacciones aquellos que tienen las ideas novedosas más útiles; las conversaciones con otros colegas multiplican las ideas creativas (Lehrer, 2012: 201). Para Csikszentmihalyi (1998: 23), «los centros de creatividad tienden a estar en la intersección de culturas diferentes», pues la creatividad supone cruzar las fronteras de los campos, interactuar con otros creadores.

Por otra parte, frente a la premisa en la que se basa la técnica de la lluvia de ideas (la crítica coarta la creatividad), estudios posteriores muestran las limitaciones de esta técnica frente a la importancia del debate en el surgimiento de nuevas ideas (Lehrer, 2012: 208).

Por todo ello, en las asignaturas es necesario crear un ambiente de intercambio entre los estudiantes, manteniendo sesiones de taller, potenciando las intervenciones y la revisión entre los alumnos tanto en clase como a través de la plataforma virtual. Cualquier profesor de taller sabe de la importancia que el intercambio personal, no solo vertical sino horizontal, tiene en la potenciación de la creatividad. Igualmente, se ha de buscar en los temas un continuo diálogo con otras disciplinas artísticas, sociales o científicas.

\section{LA ESCRITURA CREATIVA COMO ITINERARIO ESPI- RITUAL Y COMO OFICIO}

En su estudio sobre la influencia de los programas de EC en la narrativa norteamericana, McGurl (2009: 34) cita tres lemas que históricamente han simbolizado la pedagogía de las etapas principales de la enseñanza de esta materia (marcando la poética implícita de los mismos): "write what you know», que se remonta al menos al siglo XIX y se vincula a la experiencia del autor; "show, don't tell», que encuentra difusión a principio de los años veinte y centra su atención en el oficio; "find your voice», que se origina en los sesenta, en una pedagogía que trata de acentuar lo puramente creativo. Mientras que el primero y el tercero ponen su énfasis en la expresión del yo del autor, el segundo se centra en la técnica. Básicamente estos tres lemas siguen presentes en la enseñanza actual de la EC, en diversa proporción según la poética (implícita o explícita) adoptada por el profesor.

Myers (2006: 14), en su historia sobre la enseñanza de la EC, observa cómo en sus orígenes esta materia se insertaba dentro de una educación humanística cuyo objetivo era la mejora de los alumnos como seres humanos. A finales del XIX, durante el auge de la asignatura English composition, Barrett Wendell centró su dedicación en el cultivo de la percepción, con el objetivo de que los estudiantes desarrollaran determinados hábitos mentales esenciales para la literatura (Myers, 2006: 51). En los años setenta, este impulso de unificación entre el arte y la vida se volcará hacia formas políticas: Boyle, por ejemplo, no pretendía formar escritores sino revolucionarios (McGurl, 2009: 223). Esta práctica continúa hasta la actualidad, a través de una figura emblemática como Wendy Bishop, que investigó los aspectos personales, terapéuticos y afectivos de la escritura, o de Vandermeulen (2005: 49), para quien escribir implica un cambio en la persona.

La institucionalización de la EC como enseñanza universitaria, que tiene lugar en lowa Writers' Workshop, se produce, por el contrario, bajo el lema del oficio, valorando, más que la auto-expresión, la disciplina, la técnica y la impersonalidad (McGurl, 2009: 147).

Esta dialéctica parece reproducir la que tiene lugar entre los creadores que defienden el trabajo y la técnica como fundamento de su labor literaria y aquellos que cifran lo principal de su tarea en un estado, inspiración o proceso inconsciente. Sin embargo, como hemos visto, la creatividad abarca distintas etapas y ha de ser trabajada de un modo integral. No se puede romper (Marina, 1995: 192) el lazo que une las actividades cotidianas de la inteligencia con el arte o la ciencia ${ }^{3}$. Podríamos citar algunos ejemplos (del ámbito teatral en este caso) que muestran cómo para los creadores la literatura es una vía de conocimiento y profundización en la interioridad: las técnicas de Mijail Chéjov, influidas por la antroposofía de Steiner (Ruiz, 2012: 147); el fin de la representación según Beck -«busca de una condición de conciencia que (...) acerca más a Dios» (Ruiz, 2012: 313)-; la superación de las opresiones internas en Boal, algunas de cuyas técnicas se emplean en el teatro terapéutico (Fernández y Montero: 2012); el teatro pobre de Grotowski (con su concepción del actor santo) o su para-teatro (Ruiz, 2012: 366).

La integración del hombre actúa, como es sabido, sobre tres ámbitos: consigo mismo, con el otro, con la naturaleza. Hemos mencionado brevemente la integración de la persona consigo misma y en el punto 7 nos extenderemos sobre la dialéctica entre escritor y sociedad; debemos mencionar, siquiera de una manera somera, la necesaria sutura de la escisión entre el hombre y la naturaleza. En un ámbito cada vez más dominado por lo virtual, la naturaleza nos sigue llamando con su belleza y su misterio. Contemplarla nos devuelve a lo concreto y a lo real (Burnside, 2006: 93), al tiempo que nos convoca a su cuidado. El alumno ha de avanzar en el asombro (fuente de la poesía y de la ciencia) como modo de unificar estos ámbitos 
del conocimiento y como modo de restituir una relación respetuosa con la naturaleza. Durante el curso, en alguna de las asignaturas puede dedicarse alguna acción docente a este fin, como las planteadas por Gorrell (2011: 231-252).

\section{ESCRITURA CREATIVA COMO REINTEGRACIÓN DE LOS ESTUDIOS LITERARIOS}

Aunque esta disciplina conlleve cambios en la docencia y nuevos frentes en la investigación, no se trata de crear una nueva rama, ni de establecer nuevas divisiones. Al contrario, la EC ha de aunar conocimientos de historia de la literatura, teoría y crítica literaria, pero poniendo el énfasis en la competencia generadora. En este sentido, Foerster considera que quien quiera ser serio en literatura necesita una familiaridad íntima con las tradiciones de las artes, una educación literaria más a fondo y el desarrollo de «a whole set of powers that should be active in the study of literature. It means the development not only of a [philological] sense of fact and a [historical] sense of time (...) but it means also the development of aesthetic responsiveness, of the ability to handle ideas, of taste and judgement or the critical sense, and the power of writing and speaking in the sensitive language appropiate to a literary discussion» (Myers, 2006: 132-133). Por todo ello, la enseñanza de la EC no puede limitarse a simple taller.

\section{DOS FUNDAMENTOS DEL OFICIO OLVIDADOS: IMI- TACIÓN Y RETÓRICA}

A partir del siglo XVIII, la modernidad viene a barrer las tres formas de imitación que habían recorrido la historia de la cultura y el pensamiento desde los griegos (imitación estética de la naturaleza, imitación metafísica de las ideas, imitación técnica-retórica de los antiguos). El arte no se sustenta en la naturaleza, sino en la genialidad del creador y en el placer del espectador; las ideas son sustituidas por la mente del sujeto; el pasado se reemplaza por la idea de progreso. El origen de este cambio radica en la sustitución del centro de la cultura anterior (modelo-copia) por un nuevo centro, el sujeto moderno (Gomá, 2014: 229). Pero este sistema (en su raíz solipsista) no sirve para comprender los procesos humanos (en los que lo personal se entrelaza con lo comunitario y se enraíza en una tradición), ni el proceso sistémico de la creatividad, ni la enseñanza de la escritura. De hecho, termina con uno de los pilares del aprendizaje literario (y humano), sin ofrecer ningún otro modelo (simplemente repitiendo que «no se puede enseñar a escribir»).
Sin embargo, desde el final del XIX se han alzado voces que, a partir de diversas disciplinas, han reivindicado una nueva comprensión de la imitación y su necesidad en el aprendizaje (Gomá, 2014): los estudios de Piaget sobre la imitación en el surgimiento de la inteligencia del niño, así como la persistencia en la madurez de figuras ejemplares; los de Scheler sobre la "coejecución de los actos» por el amor; estudios sobre la estructura imitativa de la sociedad (de Bagehot o Tarde); la repetición del arquetipo en Eliade; la reivindicación de la mímesis artística en Adorno; el entrecruzamiento de deseo y mímesis (Girard); los tres momentos de la Mímesis en Ricoeur; y, en el ámbito de la imitación de textos, los estudios de Genette sobre transtextualidad en Palimpsestos.

De hecho, en la práctica de la escritura ha seguido manteniéndose de un modo intermitente la técnica de la imitación y en la actualidad comienza a ser defendida de nuevo. Así se observa en la obra de Hunley (2007: 91), que considera que el camino mejor para desarrollar un estilo original es a través de la imitación deliberada de elementos estilísticos de poetasmodelo y propone actividades concretas que realiza en sus clases. En el ámbito francófono, Houdart-Merot defiende la reescritura y la imitación (reinterpretación o transformación textual) como métodos principales de enseñanza en su libro Réécriture et écriture d'invention au lycée.

El estilo de un escritor se forma a partir de la lectura y el diálogo con otros escritores, que se van asimilando (o rechazando), aunque ni en una etapa de madurez se deja de tener modelos. Necesariamente, las asignaturas habrán de plantear una serie de textos que sirvan de modelo para el aprendizaje de los alumnos: modelos genéricos, estilísticos, retóricos. Es necesario un análisis detenido y la posterior ejecución de un texto que muestre la asimilación de los componentes estudiados.

Dado que la imitación presupone un canon (problema para la cultura posmoderna), partamos para la definición de canon de la propuesta por Sullà (1998: 11): «una lista o elenco de obras consideradas valiosas y dignas de ser estudiadas y comentadas». En el incierto presente, investigadores, editores y críticos realizan una selección, de entre la cual (conviene no obviarlo) el profesor ha de escoger. El elenco de textos refleja, por una parte, una comprensión histórica y, por tanto, inestable y dialécticamente configurada, de la literatura contemporánea (Pozuelo Yvancos, 1998: 226); por otra parte, se basa en una concepción teórica que lo organiza (la literatura como forma de conocimiento que 
se vierte en géneros, que se concretan en elementos compositivos que son herramientas de comprensión).

El modelo propuesto ha de ser un prototipo, es decir, una obra concreta pero que pueda representar un colectivo (y, más aún, que pudiera ser universal): reúne características particulares, pero se ha erigido en ejemplo de otros autores (siendo representativo de un determinado género, estilo o tendencia) y muestra un ideal de excelencia (Gomá, 2014: 500) a través de la belleza, entendida como revelación de la verdad (epifanía que causa algún aspecto de la realidad en su aparecer). El prototipo ha de ser revelación de algún ámbito de la vida. La aparición de esta verdad se impone y engendra el deseo de seguir el modelo para alcanzar el prototipo que se esconde tras él. Por ello es imprescindible seleccionar modelos que reúnan esta excelencia (capaces de cautivar al alumno lector) y, al mismo tiempo, muestren los principales modos de acercamiento a la realidad que se ofrecen en la literatura contemporánea ${ }^{4}$.

Como observa Hunley, durante la mayor parte de la historia de la civilización occidental la retórica constituyó el principal arte de composición para los poetas; desde Grecia continuó dominando la educación en la Edad Media y el Renacimiento e incluso ejerció su fuerza formadora en los siglos XVIII y XIX (Hernández Guerrero y García Tejera, 1994: 149-169). HoudartMerot (1998: 113-118) ha mostrado su influjo en la enseñanza francesa hasta los años sesenta del pasado siglo. La retórica ofrece un acercamiento sistemático a las distintas fases compositivas de un texto, adiestrando al alumno en diversas habilidades: desde la generación de la idea en la inventio o el trabajo de la forma (dispositio y elocutio) hasta la práctica de aspectos esenciales y por desgracia preteridos hoy como son la ejercitación de la memoria y la actio.

Aunque, desde una perspectiva individualista o heredera aún del genio romántico, se crea que el límite reseca o mata la creatividad, por el contrario se revela fecundo: los estudios muestran (Abraham, 2018: 205) que las constricciones externas mejoran la creatividad, hasta el punto de que en ciertos ejercicios de escritura creativa se consiguen mejores resultados (un uso más complejo del lenguaje) con unas instrucciones limitadoras que sin ellas.

\section{ESCRITURA CREATIVA Y SOCIEDAD: SALIR DE LA BURBUJA}

En su libro sobre la influencia de los programas de EC en la narrativa norteamericana, McGurl sostiene que los programas han fomentado una «auto-poética de la auto-referencialidad». Los programas serían el reflejo de una modernidad reflexiva, potenciando la reflexión de los escritores sobre su propio yo y sobre el propio proceso de la escritura. Participan así los programas en una dinámica social de auto-observación, en la que el individuo siente una compulsión por el auto-diseño o la puesta en escena de sí mismo a través de una biografía (McGurl, 2009: 12). De ahí la fuerte tendencia metaliteraria de los principales subgéneros surgidos o desarrollados en los programas de EC. Si bien el origen de este impulso metaliterario ha de hallarse precisamente en la modernidad, es un hecho que los talleres refuerzan la auto-observación y la reflexión sobre el proceso de escritura.

Dawson (2005: 87-120) propone reemplazar esta poética de talleres por una poética sociológica, en la cual la escritura no sea una práctica individual apartada de la sociedad, y el análisis en clase entre también en las implicaciones políticas y sociales, consciente (desde una perspectiva bajtiniana) del choque entre distintos discursos que viven en la sociedad y se plasman en la polifonía de las obras. Este riesgo de las asignaturas de escritura ha sido contrarrestado en la práctica por algunos profesores que la imparten, que han tratado de buscar soluciones que hagan salir a los alumnos de un entorno cerrado (Manolis, 2005).

En conclusión, si la literatura es una forma de conocimiento, no puede vivir de espaldas al otro, ha de buscar el contacto con una sociedad que va más allá del propio grupo de alumnos escritores. En este sentido será necesario habilitar determinadas metodologías que ejerciten en el alumno una apertura y sensibilidad hacia entornos sociales distintos del suyo propio. En la línea de Manolis, resulta positivo que los alumnos tengan alguna experiencia de marginalidad real que los interpele.

Finalmente, las asignaturas no han de perseguir simplemente acciones aisladas, sino que, al proponer actividades en grupo y proyectos de mayor duración (voluntariado, recitales), procuran establecer redes estables que, con una vinculación débil con la institución (pues el objetivo es la autonomía del alumno), puedan proseguir al término del curso. A esto se animará durante el desarrollo de las sesiones, por el carácter social que tiene el proceso creativo: este no solo atañe a los textos, sino que también elabora un mundo de sentido a través y a lo largo de las relaciones vividas en estas experiencias (O'Rourke, 2005: 57). De este modo, promoviendo el surgimiento de pequeñas comunidades de escritores que interaccionen según sus propios intereses, involucrándose en 
algunos casos en la actividad cultural de la ciudad, la universidad contribuye modestamente a la creación del tejido literario de la comunidad.

\section{INVESTIGACIÓN EN ESCRITURA CREATIVA}

Aunque se trate de un terreno espinoso, no podemos concluir el artículo sin abordar la necesidad de un desarrollo de la investigación en EC. El dominio de la EC como disciplina académica es complejo, disputado, y se encuentra aún por fundar. Esta situación se produce incluso en Estados Unidos, donde la materia lleva más de un siglo impartiéndose, lo que da muestra de la dificultad que entraña la empresa. Bizzaro (2004: 300) trata de establecer un ámbito propio, pero reconociendo que aún no existe. Lim (2003: 151) formula una pregunta que todavía no tiene respuesta definitiva: "How does the modern research university incorporate or contain creative writing?». Mayers (2009: 217) especifica aún más esta cuestión: «ls it possible to refer to such a thing as "creative writing studies"? And if the answer is yes, then what does the term mean?». Aún en 2012 Donnelly dedica una monografía precisamente a este fin: Establishing Creative Writing Studies as an Academic Discipline.

En un libro anterior, Mayers (2005: XIV) defiende un tipo de escritura que denomina "craft criticism», en la que ve la posibilidad de una investigación teórica en EC (Mayers, 2005: 34): en ella, la preocupación por la producción textual toma precedencia sobre cualquier preocupación por la interpretación y suele tener un elemento pedagógico o evaluativo (cómo se puede mejorar la escritura). Mayers parece incentivar los rasgos reflexivos y críticos propios del escritor y de su intervención en el mundo literario, aunque institucionalizados por su nueva posición (en tanto que comunidad científica) en los departamentos universitarios.

Sin embargo, en el artículo posterior ya citado (2009), Mayers trata de establecer el ámbito de los estudios de EC, llegando a conclusiones diversas de las de su libro (Re)Writing Craft. Tras distinguir la empresa educativa de la EC de los estudios de EC, define a estos (Mayers, 2009: 218), citando a Haake, como una investigación que «seek[s] to move us beyond our preoccupation with the writer and the text to the role of creative writing as an academic discipline inside a profession that includes, but is not limited to, the production and teaching of imaginative writing».

Se aprecia en la pasada definición cómo Mayers trata de crear un espacio propio para la EC separándose de los estudios literarios y de composición. En el ar- tículo distingue Mayers (2009: 220) tres ramas principales en los estudios de EC: pedagógica, histórica y apologética. En efecto, el origen de estos estudios como literatura científica claramente diferenciada se origina en un intento de responder a una serie de problemas pedagógicos surgidos con el desarrollo de la enseñanza de esta materia y encuentra en Wendy Bishop a su pionera. Para afrontar, sin embargo, la crisis en la pedagogía, los investigadores volvieron sus ojos a la historia tratando de encontrar caminos en esta crisis. La tercera línea de los estudios sería la apologética (la defensa del valor de la propia materia). Mayers (2009: 221) también alude a una rama teórica, constituida por trabajos en los que el escritor se pregunta cómo se produce un texto concreto, si bien llega a encontrar en algunos textos de poética (como «The Philosophy of Composition» de Poe) un antecedente de los estudios de EC.

Bizzaro ha tratado de diferenciar la EC de otras áreas típicamente asociadas con los estudios ingleses, convencido de que, aunque algunas competencias evaluadas por otras ramas de los estudios literarios son las mismas que las empleadas en EC, algunos usos de esas competencias son absolutamente únicos en el nuevo campo, pues difieren en un nivel epistemológico: la investigación desde la perspectiva del escritor, la importancia de la historia en la creación, la comprensión del lector, la fe en el proceso de creación. Por ello concluye afirmando el surgimiento de una nueva disciplina, debido a su diferencia epistemológica con otras áreas (Bizzaro, 2004: 308).

El esfuerzo emprendido en las universidades americanas para desligar por completo los estudios creativos se funda en algunas razones de carácter científico como las enunciadas, pero está potenciado por una situación académica que favorece la autoafirmación: la lucha por un terreno propio, por una independencia académica. Dado que el surgimiento de esta disciplina tuvo lugar en pugna con los otros estudios literarios (en una relación que osciló entre la ignorancia y el desprecio por parte de los viejos estudios), los profesores de EC que han querido forjar una carrera académica han debido buscar nuevas plataformas (revistas, congresos, asociaciones) para sus trabajos.

Si bien la EC abre nuevos ámbitos de conocimiento, en otros casos revaloriza prácticas tradicionales de los profesores-escritores o enfoques de la investigación que habían sido preteridos por el positivismo, el inmanentismo o la hipertrofia teórica postestructuralista: el ensayo, la crítica, las investigaciones sobre el proceso de escritura, la perspectiva del autor en el estudio 
de lo literario ${ }^{5}$. De esta manera han de entenderse algunos textos escritos por poetas profesores como los ensayos de García Montero ${ }^{6}$ o los diarios de Sánchez Robayna. Estas líneas de investigación han de reconocerse y defenderse, ya que, de lo contrario, los cada vez más frecuentes filtros que se nos presentan como presuntamente objetivos (rankings de editoriales y factores de impacto) terminarán por expulsar prácticas tradicionalmente humanistas y literarias.

Más aún, hay que reconocer que las investigaciones pertenecientes al ámbito de la EC se fundan en una perspectiva distinta a la historicista (de ahí que se hayan ligado más al ámbito de la crítica), persiguen objetivos diferentes (cómo se produce un buen texto) y por ello pueden emplear distinta metodología. Pero, de nuevo, estas preguntas abren a nuevos campos al tiempo que se enraízan en disciplinas de larga tradición (como son la retórica y la poética). Obsérvese, por ejemplo, el seminal libro de Javier Blasco (2011), en el que fija como objetivo de la crítica genética el estudio del proceso creativo, indicando una metodología de acceso a las diversas fases de este proceso.

Como se afirmó al principio de este trabajo, no parece conveniente una mayor subdivisión en los ámbitos literarios; como Foerster, consideramos estos estudios como una parte de la tradición literaria que vuelve a incorporar ramas que habían sido desgajadas del tronco de la literatura, debido a una concepción en exceso «cientificista» que ignoraba la vertiente productiva del hecho literario.

Finalmente, en la reflexión sobre el establecimiento de un dominio de la EC, los trabajos norteamericanos olvidan con frecuencia una dimensión esencial: la consideración del propio trabajo creador como investigación. En los últimos años, la crítica australiana ha avanzado buscando una fundamentación metodológica a este strange loop que supone siempre la relación entre teoría y práctica de la EC (Boyd, 2009: 6). Así Stewart (2003) defiende la necesidad de enfocar en la propia práctica la exploración de ideas para la elaboración de una teoría que se interrelacione con la actividad creadora. La diferencia entre el creador universitario y el que no pertenece a este ámbito se funda en que al primero se le ha de demandar una conciencia de los presupuestos, procesos y percepciones de su práctica artística, de modo que pueda exponerlos teóricamente y servirse de este conoci- miento para avanzar en su materia. El investigador ha de exponer sus creaciones para demostrar el tipo de conocimiento generado por su actividad en su campo. También Cook (2005) defiende la escritura como un método de investigación.

En Francia (Houdart-Merot, 2018: 104), los distintos tipos de doctorado relacionados con el ámbito de la $E C$, «reposent toutes sur la conviction que la création est en elle-même une recherche, qu'elle peut se penser et que ce savoir de la création et sa théorisation méritent d'entrer à l'université». Creación y reflexión teórica se entrecruzan en un proceso recurrente. Es la misma convicción que guía los trabajos de fin de máster (y alguna tesis) que se presentan en España.

A pesar de estos avances, las investigaciones en otras artes pueden abrir nuevas vías metodológicas para el ámbito de la EC. Como ha observado Hannula, Suoranta y Vadén (2014: XI), los investigadores artísticos tienen al menos tres trabajos que afrontar: desarrollar y perfeccionar sus propios oficios artísticos, su pensamiento conceptual y creativo haciendo arte y conceptualizándolo; presentar sus avances ante la comunidad académica, proponiendo de forma argumentada su teoría; comunicarse con otros artistas y con un público más extenso. La investigación artística constaría de dos pilares (Hannula, Suoranta y Vadén 2014: 15): "artistic research = artistic process (acts inside the practice) + arguing for a point of view (contextual, interpretative, conceptual, narrative work)». Tanto la acción como la argumentación y la hermenéutica son imprescindibles.

Esta investigación creadora debería constituir uno de los objetivos de la EC en la universidad, pues está lejos de ser aceptada como investigación en nuestro ámbito (al contrario del ámbito anglosajón o del de las bellas artes), aunque, como apuntábamos antes, los escritores que a la vez son profesores realizan en sus textos con frecuencia esta búsqueda. El creador ha de estar en continua formación y contacto con las palabras para lograr que nuevas experiencias o ámbitos diferentes de la realidad se revelen en la escritura; esta búsqueda se nutre de sus investigaciones y repercute de igual modo en la docencia, enriqueciéndola. De este modo, al devolver a la palabra su poder creador, se sutura la escisión a la que la herencia de cierta modernidad ha condenado a los estudios literarios. 


\section{NOTAS}

1. Cfr. el estudio sobre las técnicas de mindfulness en la enseñanza de EC (Vázquez Medel, 2014).

2. Para un resumen, cfr. Romo, SanchezRuiz y Alfonso-Benlliure (2017)

3. Como nos recuerda la cita de Hölderlin («Dichterisch wohnet der Mensch»).

\section{REFERENCIAS}

Abbs, Peter (1989). A is for Aesthetic. Essays on Creative and Aesthetic Education. New York: The Falmer Press.

Abraham, Anna (2018). The Neuroscience of Creativity. Cambridge: Cambridge University Press. https://doi. org/10.1017/9781316816981

Andreasen, Nancy C. (2005). The Creating Brain: The Neuroscience of Genius. New York: Dana Press.

Baas, Matthijs; Roskes, Marieke; Sligte, Daniel; Nijstad, Bernard A. y De Dreu, Carsten K. W. (2013). Personality and Creativity: The Dual Pathway to Creativity Model and a Research Agenda. Social and Personality Psychology Compass, 7 (10): 732-748. https://doi. org/10.1111/spc3.12062

Barash, Jeffrey Andrew (1994). Symbolic Construction of Reality. Chicago: University of Chicago Press.

Barbot, Baptiste; Tan, Mai; Randi, Judi; Santa-Donato, Gabrielle y Grigorenko, Elena L. (2012). Essential skills for creative writing: Integrating multiple domainspecific perspectives. Thinking Skills and Creativity, 7 (3): 209-223. https:// doi.org/10.1016/j.tsc.2012.04.006

Beaty, Roger E.; Benedek, Mathias; Silvia, Paul J. y Schacter, Daniel L. (2016). Creative Cognition and Brain Network Dynamics. Trends in Cognitive Sciences, 20 (2): 87-95. https://doi.org/10.1016/j. tics.2015.10.004

Beaty, Roger E.; Kenett, Yoed N.; Christensen, Alexander P; Rosenberg, Monica D.; Benedek, Mathias; Chen, Qunlin; Fink, Andreas; Qiu, Jiang; Kwapil, Thomas R.; Kane, Michael J. y Silvia, Paul J. (2018). Robust prediction of individual creative ability from brain functional connectivity. PNAS, 115 (5) 1087-1092. https://doi.org/10.1073/ pnas. 1713532115
4. Seguimos la concepción expresiva del género como modo de organizar la materia, lo cual coincide con la afirmación ción de los textos como imitación de un estilo o género.

5. Cfr. Houdart-Merot (2018: 104-126). de Genette (1989: 101) sobre la imita-

6. Cfr. la noción de ficción autobiográfica en "La poesía como género de ficción». La reconstrucción del proceso de escritura de un texto choca con la interpretación autobiográfica que el lector (conducido por el propio texto) podría realizar: no podía acercarse de esa manera al poema, al carecer de la información proporcionada en el ensayo.

Csikszentmihalyi, Mihaly (1998). Creatividad. Barcelona: Paidós.

Dawson, Paul (2005). Creative Writing and the New Humanities. New York: Routledge. https://doi. org/10.4324/9780203401019

Fernández, M.ạ Laura y Montero, Isabel (2012). El teatro como oportunidad. Barcelona: Rigden Institut Gestalt.

Gardner, Howard (2005). Arte, mente y cerebro. Barcelona: Paidós.

Blasco, Javier (2011). Poética de la escritura. Valladolid: Universidad de Valladolid.

Bono, Edward de (1994). El pensamiento creativo. Barcelona: Paidós.

Bourgeois-Bougrine, Samira; Botella, Marion; Glaveanu, Vlad; Guillou, Katell; De Biasi, Pierre Marc y Todd Lubart, Todd (2014). The Creativity Maze: Exploring Creativity in Screenplay Writing. Psychology of Aesthetics, Creativity, and the Arts, 8 (4): 384-399. https://doi. org/10.1037/a0037839

Boyd, Nicola (2009). A Creative Writing Research Methodology. En: Australasian Association of Writing Programs. The Margins and Mainstreams Papers: The Refereed Proceedings of the $14^{\text {th }}$ Conference of the Australian Association of Writing Programs. Disponible en: http://www.aawp.org.au/publications/the-margins-mainstreamspapers/ [Fecha de consulta: 20 de septiembre de 2019].

Burnside, John (2006). A Science of belonging: Poetry as Ecology. En: Crawford R. (ed.) Contemporary Poetry and Contemporary Science. Edinburgh: Edinburgh University Press, pp. 91-106.

Cook, Jon (2005). Creative Writing as a Research Method. En: Griffin, Gabriele (ed.) (2005). Research Methods for English Studies. Edinburgh: Edinburgh University Press, pp. 200-217. 
Kandel, E. R. (2013). La era del inconsciente. Barcelona: Paidós.

Kleinmintz, Oded; Goldstein, Pavel; Mayseless, Naama; Abecasis, Donna y Shamay-Tsoory, Simone, G. (2014). Expertise in Musical Improvisation and Creativity: The Mediation of Idea Evaluation. PLOS ONE, 9 (7): e101568 https:// doi.org/10.1371/journal.pone.0101568

Lehrer, Jonah (2012). Imaginar. Barcelona: R.B.A. Libros.

Lim, Shirley Geok-lin (2003). The strangeness of creative writing. An institutional query. Pedagogy, 3 (2): 151-169. https://doi.org/10.1215/15314200-32-151

Manolis, Argie (2005). Writing the Community. En Anna Leahy (ed.). Power and Identity in the Creative Writing Classroom. Clevedon: Multilingual Matters Ltd., pp. 141-151.

Marina, José Antonio (1995). Teoría de la inteligencia creadora. Barcelona: Anagrama.

Marina, José Antonio y Marina, Eva (2013). El aprendizaje de la creatividad. BarceIona: Ariel.

Marina, José Antonio y Pombo, Álvaro (2013). La creatividad literaria. Barcelona: Planeta.

Martínez-Falero, Luis (2020). Una propuesta sobre la creatividad literaria desde la Teoría de la Literatura y la Neurociencia. Archivum, LXX (I): 147-164. https://doi. org/10.17811/arc.70.1.2020.147-164

Mayers. Tim (2005). (Re) Writing Craft. Pittsburgh: University of Pittsburgh Press. https://doi.org/10.2307/j.ctt9qh4v2

Mayers, Tim (2009). One Simple Word: From Creative Writing to Creative Writing Studies. College English, 71 (3): 217-228.
McGurl, Mark (2009). The Program Era. Cambridge, Mass.: Harvard University Press. https://doi.org/10.2307/j.ctvjsf59f

Myers, David Gershom (2006). The Elephants Teach. Chicago: The Universtity of Chicago Press.

O'Rourke, Rebecca (2005). Creative Writing. Leicester: National Institute of Adult Continuing Education.

Piirto, Jane (2009). The Personalities of Creative Writers. En: Kaufman, Scott Barry y Kaufman, James C. (eds.). The Psychology of Creative Writing. New York: Cambridge University Press, pp. 3-22. https://doi.org/10.1017/ CBO9780511627101.003

Pozuelo Yvancos, José María (1998). Lotman y el canon literario. En Enric Sullà (comp.). El canon literario. Madrid: Arco Libros.

Ricoeur, Paul (2001). La metáfora viva. Madrid: Trotta.

Ricoeur, Paul (2003). Tiempo y narración. México: Siglo XXI.

Ricoeur, Paul (2011). Finitud y culpabilidad. Madrid: Trotta.

Romo, Manuela (2019). Psicología de la creatividad. Perspectivas contemporáneas. Barcelona: Paidós.

Romo, Manuela; Sanchez-Ruiz, M. José y Alfonso-Benlliure, Vicente (2017). Creatividad y personalidad a través de dominios: una revisión crítica. Anuario de Psicología, 47 (2): 57-69. https://doi. org/10.1016/j.anpsic.2017.04.003

Royster, B. (2005). Inspiration, Creativity, and Crisis: The Romantic Myth of the Writer Meets the Contemporary Classroom. En Anna Leahy (ed.). Power and Identity in the Creative Writing Classroom. Clevedon: Multilingual Matters Ltd., pp. 26-38.
Ruiz, Brent (2012). El arte del actor en el siglo XX. Bilbao: Artezblai.

Smorti, Andrea (2001). El Pensamiento Narrativo. Sevilla: Mergablum.

Stewart, Robyn (2003). (Re) inventing Artists' Research: Constructing living forms of theory. TEXT. Journal of Writing and Writing Courses, 7 (2). Disponible en http://www.textjournal.com.au/oct03/ stewart.htm [Fecha de consulta: 19 de septiembre de 2019].

Sullà, Enric (1998). El canon literario. Madrid: Arco Libros.

Torres-Irribarra, David; Ibaceta, Miguel y Preiss, David D. (2019). Positive and negative mind wandering: an assessment of their relationship with mindfulness and metacognition in university students / Divagación mental positiva y negativa: evaluando su relación con la atención plena y la metacognición en estudiantes universitarios. Studies in Psychology, 40 (3): 664-701. https://doi.org/10.1080/02 109395.2019.1679457

Vandermeulen, Carl (2005). The Double Bind and Stumbling Block: A Case Study as an Argument for Authority-conscious Pedagogy. En Anna Leahy (ed.). Power and Identity in the Creative Writing Classroom. Clevedon: Multilingual Matters Ltd., pp. 49-62.

Vázquez Medel, Manuel Ángel (2014). Creatividad y Mindfulness. En Pablo García Sempere, Pablo Tejada Romero y Ayelén Ruscica (coords.). Investigación y docencia en la creación artística. Granada: Editorial Universidad de Granada, pp. 11-27.

Wandor, Michelene (2008). The author is not dead, merely somewhere else: Creative writing after theory. Basingstoke and New York: Palgrave Macmillan.

Wilson, Edmund O. (1999). Consilience. Barcelona: Galaxia Gutenberg-Círculo de Lectores. 